\title{
Evaluation of the antifungal activity and antibiofilm of the essential oil of Ocimum canum (Alfavaca) in Candida spp.
}

\author{
Aline L. N. Marques*, Natália V. Carrasco, Sarah R. Pimenta, Giovana C. Boni, Simone N. B. de Feiria, José \\ Francisco Höfling.
}

\begin{abstract}
Essential oils from plants of the genus Ocimum have demonstrated potential for anti-Candida action, as described in the literature. The objective of the present project was to evaluate the antifungal activity against Candida spp. and the antibiofilm effect of $\mathrm{C}$. albicans of $\mathrm{O}$. canum essential oil. The essential oil showed antifungal activity between 0.0062 to $1 \mathrm{mg} / \mathrm{mL}$ and effect on metabolic activity of the biofilm by up to $50 \%$ at concentrations of $16-1 \mathrm{mg} / \mathrm{mL}$. The essential oil demonstrates antifungal activity against Candida spp. and decreases the metabolic activity of $\mathrm{C}$. albicans when in biofilm in formation and mature.
\end{abstract}

\section{Key words:}

Candida spp., Ocimum spp., Medicinal plants.

\section{Introduction}

Candida spp. fungi, related to infections associated with immunosuppressed patients, have demonstrated an increasing acquisition of resistance to the antifungal drugs available for the control of these microorganisms, which has led the researchers of this area to look for new alternatives of drugs that have an action against these organisms. In this context, essential oils extracted from plants of the genus Ocimum have demonstrated the potential of antimicrobial action, especially antifungal activity, against fungi of the species Candida, as described by several authors in the scientific literature. Therefore, the present project aimed to evaluate the antifungal activity against Candida spp. and the biological effect of the essential oil on biofilm formation by Candida albicans (SC 5314).

\section{Results and Discussion}

The antimicrobial effect was evaluated by the broth microdilution assay against 16 strains of Candida spp. (CSLI, M27-A3, 2008). The biological effect of the essential oil on the biofilm was evaluated in the biofilm in formation and mature. From an overnight culture, the inoculum was adjusted to $1 \times 10^{6} \mathrm{cell} / \mathrm{mL}$ and both were pre-incubated, being a pre-incubation of $2 \mathrm{~h}$ (biofilm formation) and another of $24 \mathrm{~h}$ (mature biofilm) respectively. After this period both biofilms were treated and incubated for $24 \mathrm{~h}$. The biofilms were quantified after $2 \mathrm{~h}$ exposure of $80 \mu \mathrm{l}$ of XTT and read in a microplate spectrophotometer at $490 \mathrm{~nm}$.

The essential oil of Ocimum canum showed antifungal activity between $0.0062-1 \mathrm{mg} / \mathrm{mL}$. The lowest concentration found was for $C$. utilis.

The oil showed an effect on the decrease of the metabolic activity of $C$. albicans in biofilm formation and mature biofilm in $50 \%$ between concentrations of $16-1$ $\mathrm{mg} / \mathrm{mL}$.
Image 1. Ocimum canum oil against Candida albicans biofilm

Ocimum canum oil against Candida albicans biofilm

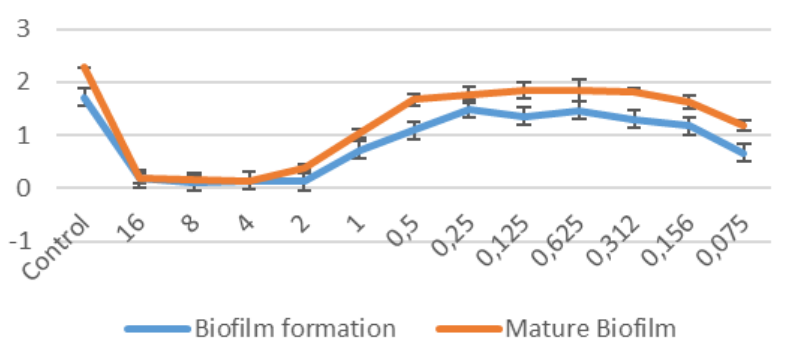

Chart 1. Determination of minimum inhibitory concentration (MIC)

\begin{tabular}{|c|c|c|c|}
\multicolumn{1}{c|}{ CEPA } & [ ] $\mathrm{g} / \mathrm{mL}$ & \multicolumn{1}{c|}{ CEPA } & [ ] $\mathrm{g} / \mathrm{mL}$ \\
\hline CA SC 5314 & 1 & CL ATCC 4031 & 1 \\
\hline CA ATCC 90028 & 1 & CL IZ06 & 0,5 \\
\hline CA CBS 562 & 1 & CP ATCC 22019 & 0,25 \\
\hline CD CBS 7987 & 0,25 & CP CBS604 & 0,25 \\
\hline CG IZ07 & 1 & CR IZ12 & 0,5 \\
\hline CGUI CBS 566 & 0,25 & CT ATCC 40281 & 0,5 \\
\hline CK ATCC 6258 & 1 & CT CBS 94 & 0,5 \\
\hline CK CBS 573 & 0,5 & CUT CBS 560 & 0,0062 \\
\hline
\end{tabular}

CA: C. albicans; CD: C. dubliniensis; CG: C. glabrata; CGUI: C guilliermondii; CK: C. krusei; CL: C. lusitanae; CP: C. parapsilosis; CR: $C$ rugosa; CT: C. tropicalis; CUT: C. utilis;

\section{Conclusions}

The essential oil of Ocimum canum demonstrates antifungal activity against Candida spp. and decreases the metabolic activity of $C$. albicans when in biofilm. 